\title{
TWO FAMILIES OF FLAG-TRANSITIVE AFFINE PLANES
}

Dedicated to Otto Wagner on the occasion of his 60th birthday

ABSTRACT. Two families of flag-transitive nondesarguesian affine planes of odd order are defined, and isomorphisms among the various planes are studied.

Thirty years ago Wagner [5] proved the important result that every finite flag-transitive affine plane is a translation plane. However, since that time relatively few classes of such planes have been found. The purpose of this note is to comment on two constructions of flag-transitive affine planes due to Suetake [4]. These constructions will be generalized slightly and will be shown to produce fairly large numbers of pairwise nonisomorphic planes. Most of them are new (compare [1], [4] and the references therein), but those that are 2-dimensional over their kernels were known previously [1], [2].

Let $F=\mathrm{GF}\left(q^{2 n}\right), L=\mathrm{GF}\left(q^{n}\right)$ and $K=\mathrm{GF}(q)$, where $n>1$ and $q=p^{e}$ is a power of an odd prime $p$. Let $\sigma \in \operatorname{Gal}(L / K)$; we will assume (unless stated otherwise) that $\sigma \neq 1$. Throughout this note we will identify each such automorphism $\sigma$ with a power of $q$ inducing it.

Assume that $n$ is odd. Let $b \in F$ be such that $\bar{b}=-b$ (where bar denotes the involutory automorphism of $F$ ), so that $b^{2}=-b \bar{b} \in L$. Let $s \in F^{*}$ have order $\left(q^{n}+1\right)(q-1)$. Write $h(x)=x+b x^{\sigma}$ for $x \in F$, and consider the set

$$
\mathscr{S}_{b, \sigma}=\left\{s^{i} h(L) \mid 0 \leqslant i \leqslant q^{n}\right\}
$$

of subspaces of $F$, where $F$ is always viewed as a $K$-space. This is very slightly more general than Suetake's construction (he assumes that $\sigma=q$ and $\left.q^{n} \equiv 3(\bmod 4)\right)$.

For $\alpha \in F$ let $\tilde{\alpha}$ denote the linear transformation $z \rightarrow \alpha z$ from $F$ to itself.

THEOREM 1. (i) $\mathscr{S}_{b, \sigma}$ is a spread, and defines a nondesarguesian translation plane $\Pi_{b, \sigma}$.

(ii) $\Pi_{b, \sigma}$ admits a flag-transitive group inducing a cyclic group on the line at infinity.

(iii) The number of pairwise nonisomorphic translation planes arising in this

*Research supported in part by NSF grant DMS 87-01794 and NSA grant MDA 904-88-H2040 .

Geometriae Dedicata 41: 191-200, 1992.

(C) 1992 Kluwer Academic Publishers. Printed in the Netherlands. 
manner is at least $\frac{1}{2}\left(n / n^{*}-1\right)\left(q^{n^{*}}-1\right) / 2 e n^{*}$, where $n / n^{*}$ is the smallest prime factor of $n$.

Proof. (i, ii) First of all, $h$ is injective: if $x+b x^{\sigma}=0$ with $x \in L$ then also $x+\bar{b} x^{\sigma}=0$, so that $x=0$. Thus, $\mathscr{S}_{b, \sigma}$ consists of $n$-dimensional $K$-spaces.

Clearly, $\tilde{s}$ induces a cycle transitive on $\mathscr{S}_{b, \sigma}$, so that (ii) holds if (i) is presupposed.

Assume that $h(x)=s^{i} h(y)$ with $x, y \in L^{*}$ and $0<i \leqslant q^{n}$. Then

$$
\left(x+b x^{\sigma}\right)\left(x+\bar{b} x^{\sigma}\right)=s^{i} \bar{s}^{i}\left(y+b y^{\sigma}\right)\left(y+\bar{b} y^{\sigma}\right),
$$

so that $x^{2}-b^{2}\left(x^{\sigma}\right)^{2}=k\left[y^{2}-b^{2}\left(y^{\sigma}\right)^{2}\right]$ with $k=s^{i} s^{i} \in K$. Then $x^{2}-k y^{2}=b^{2}\left(x^{2}-k y^{2}\right)^{\sigma}$. However, $\left(x^{2}-k y^{2}\right)^{\sigma-1}$ is the square of an element of $L$ whereas $b^{2}$ is not. Consequently, $x^{2}-k y^{2}=0$, so that $x=m y$ where $m \in L$ and $m^{2}=k$. Since $n$ is odd, so is $\left(q^{n}-1\right) /(q-1)$, and hence $m \in K$. Then $m\left(y+b y^{\sigma}\right)=m y+b(m y)^{\sigma}=s^{i}\left(y+b y^{\sigma}\right)$, so that $m=s^{i},\left(s^{i}\right)^{q-1}=1$, and hence $\left(q^{n}+1\right)(q-1) \mid i(q-1)$, whereas $0<i \leqslant q^{n}$. This contradiction proves (i).

Before proving (iii) we will need an isomorphism criterion. Let $H(\sigma)=\left\{z \rightarrow \alpha^{1-\sigma} z^{\varphi}\right.$ for $z \in F \mid \alpha \in L^{*}, \varphi \in$ Aut $\left.F\right\}$, so that $H(\sigma)$ induces a group of permutations of $b L^{*}=\left\{d \in F^{*} \mid \bar{d}=-d\right\}$. Note that $|H(\sigma)|=2 \operatorname{en}\left(q^{n}-1\right) /\left(q^{n}-1, \sigma-1\right)$.

LEMMA 1. (I) $\Pi_{b, \sigma} \cong \Pi_{b^{-1}, \sigma^{-1}}$.

(II) If $\Pi_{b, \sigma} \cong \Pi_{c, \tau}$ then $\tau=\sigma^{ \pm 1}$.

(III) $\Pi_{b, \sigma}$ is nondesarguesian.

(IV) $\Pi_{b, \sigma} \cong \Pi_{c, \sigma}$ if and only if $b$ and $c$ are in the same $H(\sigma)$-orbit.

Proof. The transformation $z \rightarrow b^{-1} z$ sends $x+b x^{\sigma}$ to $x^{\sigma}+b^{-1}\left(x^{\sigma}\right)^{\sigma^{-1}}$, so (I) holds.

If $b$ and $c$ are in the same $H(\sigma)$-orbit then $c=\alpha^{1-\sigma} b^{\varphi}$ for some $\alpha \in L$ and $\varphi \in$ Aut $F$. Then $\left(\alpha y^{\varphi}\right)+c\left(\alpha y^{\varphi}\right)^{\sigma}=\alpha\left(y+b y^{\sigma}\right)^{\varphi}$, so that the transformation $z \rightarrow \alpha z^{\varphi}$ induces an isomorphism $\Pi_{b, \sigma} \cong \Pi_{c, \sigma}$, which proves part of (IV). We will prove (II), (III) and the harder part of (IV) simultaneously.

Note that $\mathscr{S}_{c, \tau}$ and $\Pi_{c, \tau}$ are also meaningful when $\tau=1-$ in which case $\Pi_{c, \tau}$ is desarguesian (since then $\left.x+c x^{\tau}=(1+c) x\right)$. With this in mind, assume that $\Pi_{b, \sigma} \cong \Pi_{c, \tau}$, where we temporarily allow the possibility that $\tau=1$. Note that $K$ is contained in the kernel of both of these planes. (Recall that the kernel of $\Pi_{b, \sigma}$ is the field consisting of all endomorphisms of the abelian group $F$ that map each line through 0 into itself.) It follows that there is a $K$ semilinear transformation $g: F \rightarrow F$ sending $\Pi_{b, \sigma}$ to $\Pi_{c, \tau}$ and hence sending $\mathscr{S}_{b, \sigma}$ to $\mathscr{S}_{c, \tau}$.

Let $s_{0} \in\langle s\rangle$ have prime order and generate $F^{*}\left(s_{0}\right.$ exists by [6]). Then a 
Sylow $\left|s_{0}\right|$-subgroup of $\Gamma L(F)$ is cyclic. Clearly, $g$ conjugates the collineation group of $\Pi_{b, \sigma}$ to that of $\Pi_{c, \tau}$. By Sylow's Theorem, we may assume that $g$ conjugates $\left\langle\tilde{s}_{0}\right\rangle$ to itself. Then $g$ has the form $z \rightarrow \alpha z^{\varphi}$ with $\alpha \in F^{*}$ and $\varphi \in$ Aut $F$ (for all $z \in F$ ). Since $\Pi_{b, \sigma} \cong \Pi_{b^{q}, \sigma}$ (by the part of (IV) already verified) and $\varphi^{-1} g$ is an isomorphism from $\Pi_{b^{\varphi}, \sigma}$ to $\Pi_{c, \tau}$, by replacing $g$ by $\varphi^{-1} g$ we may assume that $\varphi=1$. Similarly, by replacing $g$ by $g \tilde{s}^{i}$ for some $i$ we may assume that $\alpha h(L)=h(L)^{g}=h^{\prime}(L)$, where $h^{\prime}(x)=x+c x^{\tau}$ for $x \in F$.

Thus, for each $x \in L^{*}$ there is some $y \in L^{*}$ such that $x+c x^{t}=\alpha\left(y+b y^{\sigma}\right)$; and $x \rightarrow y$ defines a permutation of $L^{*}$. Then $x-c x^{\tau}=x+\bar{c} x^{\tau}=$ $\bar{\alpha}\left(y+\bar{b} y^{\sigma}\right)=\bar{\alpha}\left(y-b y^{\sigma}\right)$. Write $\quad \beta=\alpha+\bar{\alpha} \quad$ and $\quad \delta=\alpha-\bar{\alpha}$. Then $2 x=\beta y+\delta b y^{\alpha} \quad$ and $\quad 2 c x^{\tau}=\delta y+\beta b y^{\sigma}, \quad$ so that $c\{\beta y+$ $\left.\delta b y^{\sigma}\right\}^{\tau}=\delta y+\beta b y^{\sigma}$. Note that $\beta, \delta b \in L$. Then

$$
c \beta^{\tau} y^{\tau}+c(\delta b)^{\tau} y^{\sigma \tau}=\delta y+\beta b y^{\sigma} \text { for all } y \in L .
$$

Write $y=u z$ here, where $u, z \in L$. By two applications of (1),

$$
c \beta^{\tau} u^{\tau} z^{\tau}+u^{\sigma \tau}\left\{\delta z+\beta b z^{\sigma}-c \beta^{\tau} z^{\tau}\right\}=\delta u z+\beta b u^{\sigma} z^{\sigma},
$$

so that

$$
c \beta^{\tau}\left(u^{\tau}-u^{\sigma \tau}\right) z^{\tau}=\delta\left(u-u^{\sigma \tau}\right) z+\beta b\left(u^{\sigma}-u^{\sigma \tau}\right) z^{\sigma} \text { for all } u, z \in L .
$$

If $\tau=1$ then (since $\sigma \neq 1$ ) (2) implies that $c \beta=\delta$. Then $c \delta b=\beta b$ by (1), so that $c^{2} \beta=\beta$. Since $c \neq \pm 1$ it follows that $\beta=0$; but then $\delta=0$, so that $\alpha=0$, which is not the case. This proves (III). From now on we may assume that $\sigma, \tau \neq 1$.

Suppose that $\beta \neq 0$. Choose $u \in L$ not fixed by $\sigma$. Then (2) can be rewritten in the form $z^{\tau}=A z+B z^{\sigma}$ for all $z \in L$, where $A, B \in F$. In particular, $1=A+B \quad$ and $\quad\left(A u+B u^{\sigma}\right)^{2}=u^{2 \tau}=A u^{2}+B u^{2 \sigma}, \quad$ so that $\left(A^{2}-A\right) u^{2}+\left(B^{2}-B\right) u^{2 \sigma}+2 A B u u^{\sigma}=0$. Then $A B\left(u-u^{\sigma}\right)^{2}=0$, so that $A=0$ or $B=0$. It follows that either $z^{\tau}=z$ for all $z \in L$ or $z^{\tau}=z^{\sigma}$ for all $z \in L$. Consequently, $\tau=\sigma$ in this case.

Now suppose that $\beta=0$. Then $\delta \neq 0$, while (2) states that $\delta\left(u-u^{\alpha \tau}\right) z=0$. Thus, $\sigma \tau=1$ in this case.

This proves (II). We may now assume that $\tau=\sigma$.

Claim: $c \beta^{\sigma}-\beta b=0$ and $\delta=0$. Namely, (2) states that

$$
\left(c \beta^{\sigma}-\beta b\right)\left(u^{\sigma}-u^{\sigma^{2}}\right) z^{\sigma}=\delta\left(u-u^{\sigma^{2}}\right) z \quad \text { for all } u, z \in L .
$$

This proves the claim if $\delta=0$. Since $n$ is odd, $\sigma^{2} \neq 1$. If $\delta \neq 0$ then $z / z^{\sigma}$ is constant for all $z \in L^{*}$; and then $z / z^{\sigma}=1$, which is not the case.

Consequently, $c=\beta^{1-\sigma} b$ with $\beta=2 \alpha \in L$, proving the first part of (IV). 
Proof of Theorem 1 continued. (iii) Choose $\sigma$ to be one of the automorphisms $q^{j n^{*}}$ with $1 \leqslant j \leqslant \frac{1}{2}\left(n / n^{*}-1\right)$. Note that no two of these automorphisms are inverses of one another, $\left(n / n^{*}, j\right)=1$, and

$$
|H(\sigma)|=2 e n\left(q^{n}-1\right) /\left(q^{n}-1, \sigma-1\right)=2 e n\left(q^{n}-1\right) /\left(q^{n^{*}}-1\right) .
$$

For each of the $n / n^{*}$ automorphisms $\sigma^{2 i}$ with $0 \leqslant i<|\sigma|=n / n^{*}$, the stabilizer $H(\sigma)_{b}$ of $b$ contains $z \rightarrow b^{(1-\sigma) \cdot\left(\sigma^{2 i}-1\right) /(\sigma-1)} z^{\sigma^{2 i}}$, where $b^{\left(\sigma^{2 i}-1\right) /(\sigma-1)} \in L$ since $\left(\sigma^{2 i}-1\right) /(\sigma-1)$ is even. Then the orbit $b^{H(\sigma)}$ has length $\left|b^{H(\sigma)}\right| \leqslant|H(\sigma)| /|\sigma|$, so that the number of $H(\sigma)$-orbits on $b L^{*}$ is $\geqslant\left(q^{n}-1\right) /(|H(\sigma)| /|\sigma|)=$ $\left(q^{n^{*}}-1\right) / 2 e n^{*}$. By varying $j$ we obtain at least $\frac{1}{2}\left(n / n^{*}-1\right)\left(q^{n^{*}}-1\right) / 2 e n^{*}$ nonisomorphic planes by Lemma 1.

REMARKS. 1. The kernel of $\Pi_{b, \sigma}$ is the fixed field of $\sigma$. Namely, if $K^{\prime}$ is the kernel then the $K$-space $F$ must also be a vector space over $K^{\prime}$. Then $K$ is a subfield of $K^{\prime}$, and $s_{0}$ is a $K^{\prime}$-linear transformation. It follows that $K^{\prime *}$ lies in the centralizer of $s_{0}$ in $\mathrm{GL}(F)$-i.e., in $F^{*}$.

Now if $g \in K^{*}$ then $g$ must fix the line $h(L)$ and hence, in the proof of Lemma $1, b=c$ and $g$ has the form $z \rightarrow \alpha z$ for some $\alpha \in F^{*}$. That proof yields that $b=\alpha^{1-\sigma} b$ with $\alpha \in L$, so that $\alpha$ is in the fixed field of $\sigma$. The converse is trivial.

2. We used $n^{*}$ in order to minimize the order of $H(\sigma)$. For example, if $\sigma=q^{j}$ with $(j, n)=1$ then $K$ is the fixed field of $\sigma$, so that $\left(q^{n}-1\right) /(|H(\sigma)| /|\sigma|)=(q-1) / 2 e$ and for composite $n$ we obtain significantly fewer planes than in (iii).

3. It may be instructive to compare the above construction, as well as those in [4], with one in [3]. There, spreads for flag-transitive planes consist of the following subspaces whenever $q$ is even, $n$ is odd, $r \in \mathrm{GF}\left(q^{2}\right)-G F(q)$, and $t(x)=T(x)+r x$ where $T: \mathrm{GF}\left(q^{n}\right) \rightarrow \mathrm{GF}(q)$ is the trace map: $\left\{s^{i} t(L) \mid 0 \leqslant i \leqslant q^{n}\right\}$. Moreover, $r$ and $r^{\prime} \in \mathrm{GF}\left(q^{2}\right)-\mathrm{GF}(q)$ determine isomorphic planes if and only if $r^{\prime}+1=\alpha(r+1)^{\varphi}$ with $\alpha \in \mathrm{GF}(q)^{*}, \varphi \in \operatorname{Aut~GF}\left(q^{2}\right)$.

We now turn to a second family of flag-transitive planes. Again let $F=\mathrm{GF}\left(q^{2 n}\right), L=\mathrm{GF}\left(q^{n}\right)$ and $K=\mathrm{GF}(q)$, where $n>1$ and this time $q^{n} \equiv 1(\bmod 4)$. Let $\sigma \in \operatorname{Gal}(F / K)$, and let $\left.\sigma\right|_{L}$ denotes its restriction to $L$ (note the new meaning for $\sigma$ !). Assume that $\left.\sigma\right|_{L} \neq 1$.

Let $p, e, s, b$ and $h$ be as before; write $t=s^{2}$. Set

$$
\mathscr{S}_{b, \sigma}^{\prime}=\left\{t^{i} h(L) \mid 0 \leqslant i \leqslant \frac{1}{2}\left(q^{n}-1\right)\right\} \cup\left\{t^{i} h(b L) \mid 0 \leqslant i \leqslant \frac{1}{2}\left(q^{n}-1\right)\right\} .
$$

This construction again is due to Suetake when $\sigma=q$.

THEOREM 2. (i) $\mathscr{S}_{b, \sigma}^{\prime}$ is a spread, defining a translation plane $\Pi_{b, \sigma}^{\prime}$. 
(ii) $\Pi_{b, \sigma}^{\prime}$ admits a flag-transitive group inducing a noncyclic group on the line at infinity.

(iii) If the fixed field of $\sigma$ is properly contained in $L$ then $\Pi_{b, \sigma}^{\prime}$ does not admit a cyclic group acting transitively on the line at infinity.

(iv) The number of pairwise nonisomorphic translation planes arising in (iii) is at least

$$
\begin{cases}\left(n / n^{*}-1\right)\left(q^{n^{*}}-1\right) / 4 n^{*} & \text { if } n \text { is odd and } n / n^{*} \text { is the smallest } \\ & \text { prime factor of } n \\ \left(q^{n / 2}-1\right) / e n & \text { if } n \text { is even. }\end{cases}
$$

(v) If $n$ and $q$ are primes then the number in (iv) is exactly $(q-1) / 2$.

Proof. (i, ii) As before, $\mathscr{S}_{b, \sigma}^{\prime}$ consists of $n$-dimensional $K$-spaces.

Here $\langle\tilde{t}\rangle$ has just two orbits on $\mathscr{S}_{b, \sigma}^{\prime}$. The transformation $z \rightarrow b z^{\sigma}$ sends $t^{i}\left(x+b x^{\sigma}\right)$ to $t^{i \sigma}\left(\left[b x^{\sigma}\right]+b\left[b x^{\sigma}\right]^{\sigma}\right)$ and $t^{i}\left([b x]+b[b x]^{\sigma}\right)$ to $t^{i \sigma}\left(\left[b b^{\sigma} x^{\sigma}\right]+b\left[b b^{\sigma} x^{\sigma}\right]^{\sigma}\right)$, where $b b^{\sigma} \in L$. This proves (ii) if (i) is presupposed.

As before, if $h(x)=t^{i} h(y)$ with $x, y \in L^{*}$ and $0<i \leqslant \frac{1}{2}\left(q^{n}-1\right)$, then

$$
\left(x+b x^{\sigma}\right)\left(x+\bar{b} x^{\sigma}\right)=t^{i} \bar{t}^{i}\left(y+b y^{\sigma}\right)\left(y+\bar{b} y^{\sigma}\right),
$$

so that $x^{2}-b^{2}\left(x^{\sigma}\right)^{2}=k\left(y^{2}-b^{2}\left(y^{\sigma}\right)^{2}\right)$, where this time $k=t^{i} t^{i}=\left(s^{i} \bar{s}^{i}\right)^{2}$ is a square in $K$. As before, it follows in turn that $x^{2}=k y^{2}$; that $x=m y$ with $m \in K$; that $m=t^{i}$; that $\left(t^{i}\right)^{q-1}=1$; and finally that $\frac{1}{2}\left(q^{n}+1\right)(q-1) \mid i(q-1)$, which contradicts the fact that $0<i \leqslant \frac{1}{2}\left(q^{n}-1\right)$.

In view of transitivity, it remains to consider the possibility that $h(x)=t^{i} h(b y)$ for some $x, y \in L^{*}$ and some $i$. This time

$$
\begin{aligned}
x^{2}-b^{2} x^{2 \sigma} & =\left(x+b x^{\sigma}\right)\left(x+\bar{b} x^{\sigma}\right) \\
& =t^{\bar{T}} \bar{t}^{i}\left(b y+b(b y)^{\sigma}\right)\left(\overline{b y}+\bar{b}(\bar{b} y)^{\sigma}\right)=-k\left[b^{2} y^{2}-b^{2}(b y)^{2 \sigma}\right]
\end{aligned}
$$

where $k=t^{i} t^{i}$ is a square in $K$. Then $x^{2}+k b^{2} y^{2}=b^{2}\left(x^{2}+k b^{2} y^{2}\right)^{\sigma}$, and $\left(x^{2}+k b^{2} y^{2}\right)^{\sigma-1}$ is the square of an element of $L$ but $b^{2}$ is not. Consequently, $x^{2}+k b^{2} y^{2}=0$, so that $x=m b y$ with $m^{2}=-k$. Note that $x, y \in L^{*}$ but $b \notin L$, so that $m \notin L$. On the other hand, since $q^{n} \equiv 1(\bmod 4)$ we have $-1=l^{2}$ for some $l \in L$. Then $(m l)^{2}=k$ is a square in $K$, whereas $m l \notin K$ since $l \in L$ and $m \notin L$. This contradiction proves (ii). (N.B. This argument did not use the hypothesis $\left.\sigma\right|_{L} \neq 1$. However, if $\left.\sigma\right|_{L}=1$ then $\Pi_{b, \sigma}^{\prime}$ is desarguesian.)

Once again we need an isomorphism criterion. Let $H_{1}(\sigma)=\left\{z \rightarrow \alpha^{1-\sigma} z^{\varphi}\right.$ for $z \in F \mid \alpha \in L^{*} \cup b L^{*}, \varphi \in$ Aut $\left.F\right\}$, so that $H_{1}(\sigma)$ induces a group of permutations of $b L^{*}$. Note that $\left|H_{1}(\sigma)\right|=2 e n \cdot 2\left(q^{n}-1\right) /\left(2\left(q^{n}-1\right), \sigma-1\right)$. 
LEMMA 2. (I) $\Pi_{b, a}^{\prime} \cong \Pi_{b^{-1}, \sigma^{-1}}^{\prime}$.

(II) If $b$ and $c$ are in the same $H_{1}(\sigma)$-orbit then $\Pi_{b, \sigma}^{\prime} \cong \Pi_{c, \sigma}^{\prime}$.

(III) If $\Pi_{b, \sigma}^{\prime} \cong \Pi_{c, \tau}^{\prime}$ then $\tau=\sigma^{ \pm 1}$.

(IV) If $\Pi_{b, \sigma}^{\prime} \cong \Pi_{c, \sigma}^{\prime}$ then $b$ and $c$ are in the same $H_{1}(\sigma)$-orbit.

Proof. (I) The transformation $z \rightarrow b^{-1} z$ sends $x+b x^{\sigma}$ to $x^{\sigma}+b^{-1}\left(x^{\sigma}\right)^{\sigma^{-1}}$ and $b x+b b^{\sigma} x^{\sigma}$ to $b^{-1}\left(b b^{\sigma} x^{\sigma}\right)+b^{-1} b^{-\sigma^{-1}}\left(b b^{\sigma} x^{\sigma}\right)^{\sigma^{-1}}$, where $b b^{\sigma} \in L$.

(II) If $c=\alpha^{1-\sigma} b^{\varphi}$ with $\alpha \in L^{*}, \varphi \in$ Aut $F$, then the transformation $z \rightarrow \alpha z^{\varphi}$ sends $x+b x^{\sigma}$ to $\left(\alpha x^{\varphi}\right)+c\left(\alpha x^{\varphi}\right)^{\sigma}$ and $b x+b b^{\sigma} x^{\sigma}$ to $c\left[\left(\alpha b^{\varphi} / c\right) x^{\varphi}\right]+c c^{\sigma}\left[\left(\alpha b^{\varphi} / c\right) x^{\varphi}\right]^{\sigma}$, where $\alpha b^{\varphi} / c \in L$.

If $c=\alpha^{1-\sigma} b^{\varphi}$ with $\alpha \in b L^{*}, \varphi \in$ Aut $F$, then the transformation $z \rightarrow \alpha z^{\varphi}$ sends $x+b x^{\sigma}$ to $c\left[(\alpha / c) x^{\varphi}\right]+c c^{\sigma}\left[(\alpha / c) x^{\varphi}\right]^{\sigma}$ and $b x+b b^{\sigma} x^{\sigma}$ to $\left[\alpha b^{\varphi} x^{\varphi}\right]+c\left[\alpha b^{\varphi} x^{\varphi}\right]^{\sigma}$, where $\alpha / c, \alpha b^{\varphi} \in L$.

(III, IV) As in Lemma 1, if $\Pi_{b, \sigma}^{\prime} \cong \Pi_{c \tau}^{\prime}$ then we may assume that an isomorphism is induced by a transformation $g$ of the form $z \rightarrow \alpha z^{\varphi}$ for some $\alpha \in F^{*}$ and some $\varphi \in$ Aut $F$. As before, we may also assume that $\varphi=1$, and, by using $\langle\tilde{t}\rangle$, also that $\alpha h(L)=h^{\prime}(L)$ or $h^{\prime}(c L)$. Let $\theta$ denote the automorphism $z \rightarrow \bar{z}$.

Case 1: $h^{\prime}(L)=\alpha h(L)$. As in the proof of Lemma 1, (1) and (2) hold and we have $\left.\tau\right|_{L}=\left.\sigma^{ \pm 1}\right|_{L}$. Moreover, by (I) we may assume that $\left.\tau\right|_{L}=\left.\sigma\right|_{L}$ (so that $\tau=\sigma$ or $\sigma \theta$ ). This time (2) implies that $c \beta^{\tau}-\beta b=0$, and either $\delta=0$ or $\left.\sigma^{2}\right|_{L}=1$ (where $\beta=\alpha+\bar{\alpha} \in L$ and $\delta=\alpha-\bar{\alpha}$ as before). Here $\beta^{\tau}=\beta^{\sigma}$, so that $c \beta^{\sigma}=\beta b$.

Since $g$ normalizes $\langle\tilde{t}\rangle$ it must send the $\langle\tilde{t}\rangle$-orbits on $\mathscr{S}_{b, \sigma}^{\prime}$ to those on $\mathscr{S}_{c, \tau}^{\prime}$. Thus, $\alpha h(b L)=t^{j} h^{\prime}(c L)$ for some $j$; write $w=t^{j}$. Then

$$
w\left(c x+c c^{\tau} x^{\tau}\right)=\alpha\left(b y+b b^{\sigma} y^{\sigma}\right)
$$

for another permutation $x \rightarrow y$ of $L^{*}$.

Subcase 1.1: $\left.\sigma^{2}\right|_{L} \neq 1$. We have just observed that $\delta=0$, so $\beta=2 \alpha$, and that $c=\alpha^{1-\sigma} b$ and $b$ lie in the same $H_{1}(\sigma)$-orbit. In (III) we still must show that $\tau=\sigma$, so assume that $\tau=\sigma \theta$.

Since $w\left(c x+c c^{\tau} x^{\tau}\right)=\alpha\left(b y+b b^{\sigma} y^{\sigma}\right)=c\left(\alpha^{\sigma} y\right)+c c^{\sigma}\left(\alpha^{\sigma} y\right)^{\sigma}$, it follows that $w\left(x+c^{\tau} x^{\tau}\right)=v+c^{\sigma} v^{\sigma}$, where $v=\alpha^{\sigma} y \in L$ since $\alpha \in L$. As before we find first that $\bar{w}\left(x-c^{\tau} x^{\tau}\right)=v-c^{\sigma} v^{\sigma}$, then that $k\left(x^{2}-\left(c^{\tau} x^{\tau}\right)^{2}\right)=v^{2}-\left(c^{\sigma} v^{\sigma}\right)^{2}$ where $k=w \bar{w}$ is a square in $K, c^{\tau}=\bar{c}^{\sigma}=-c^{\sigma}$ and $x^{\tau}=x^{\sigma}$, and then that $k x^{2}-v^{2}=c^{2 \sigma}\left(k x^{2}-v^{2}\right)^{\sigma}$.

Now $v^{2}=k x^{2}$ for all $x \in L$, where $v$ is an additive function of $x$ and $k$ is a square in $K$. Thus, for all $x \in L, v=m x$ for some $m \in K$. Then $m^{\sigma}=m$, and $w\left(x+c^{\tau} x^{\sigma}\right)=m x+c^{\sigma} m x^{\sigma}$ for all $x \in L$. This implies both that $w=m$ and that $w c^{\tau}=c^{\sigma} m=-c^{\tau} m$, which is ridiculous. Thus, $\tau=\sigma$.

Subcase 1.2: $\left.\sigma^{2}\right|_{L}=1$. Since $\left.\sigma\right|_{L}=\left.\tau\right|_{L} \neq 1, \sigma^{2}=\theta=\tau^{2}$ and hence $\tau=\sigma$ or $\sigma^{-1}$. This proves (III) in this situation, so by (I) we may assume that $\tau=\sigma$. Recall that $c \beta^{\sigma}=\beta b$. If $\beta \neq 0$ then $b$ and $c$ lie in the same $H_{1}(\sigma)$-orbit. 
Assume that $\beta=0$ and hence $\delta=\alpha-\bar{\alpha}=2 \alpha$. Since (1) holds with $\tau=\sigma$ we have $c \delta^{\sigma} b^{\sigma}=\delta$, so that $c \alpha^{\sigma} b^{\sigma}=\alpha$. By (3), $\quad \bar{w}\left(-c x+c c^{\sigma} x^{\sigma}\right)=$ $-\alpha\left(-b y+b b^{\sigma} y^{\sigma}\right)$, so that $(w-\bar{w}) c x+(w+\bar{w}) c c^{\sigma} x^{\sigma}=2 \alpha b y \quad$ and $(w+\bar{w}) c x+(w-\bar{w}) c c^{\sigma} x^{\sigma}=2 \alpha b b^{\sigma} y^{\sigma}$. Then

$$
\begin{aligned}
& \left\{(w+\bar{w}) c x+(w-\bar{w}) c c^{\sigma} x^{\sigma}\right\} \alpha^{\sigma} \\
& \quad=2 \alpha b \alpha^{\sigma} b^{\sigma} y^{\sigma}=\alpha b\left\{(w-\bar{w}) c x+(w+\bar{w}) c c^{\sigma} x^{\sigma}\right\}^{\sigma}
\end{aligned}
$$

for all $\quad x \in K$. Consequently, $(w+\bar{w}) c \alpha^{\sigma}=\alpha b(w+\bar{w})^{\sigma} c^{\sigma} c^{\sigma \sigma} \quad$ and $(w-\bar{w}) c c^{\sigma} \alpha^{\sigma}=\alpha b(w-\bar{w})^{\sigma} c^{\sigma}$. If $\bar{w} \neq w$ then $c=\{(w-\bar{w}) / \alpha\}^{\sigma-1} b$ with $(w-\bar{w}) / \alpha \in L^{*}$, so that $b$ and $c$ are in the same $H_{1}(\sigma)$-orbit. Suppose that $\bar{w}=w$. Then $w^{2}=w \bar{w}$ is a square in $K$, so that $w \in K$. Now $\bar{w}=w=w^{\sigma}$, so that $(w+w) c \alpha^{\sigma}=\alpha b(w+w) c^{\sigma} \bar{c}$ and hence $c \alpha^{\sigma}=-\alpha b c^{\sigma} c$. Now $-\alpha=$ $\left(\alpha^{\sigma}\right)^{\sigma}=\left(-\alpha b c^{\sigma}\right)^{\sigma}=-\alpha^{\sigma} b^{\sigma} \bar{c}=\alpha^{\sigma} b^{\sigma} c$, whereas we already knew that $c \alpha^{\sigma} b^{\sigma}=\alpha$.

Case 2: $h^{\prime}(c L)=\alpha h(L)$. This time there is a permutation $x \rightarrow y$ of $L^{*}$ such that $c x+c c^{\tau} x^{\tau}=\alpha\left(y+b y^{\sigma}\right)$. Then $-c x+c c^{\tau} x^{\tau}=\bar{\alpha}\left(y-b y^{\sigma}\right)$, so that $2 c x=\delta y+\beta b y^{\sigma}$ and $2 c c^{\tau} x^{\tau}=\beta y+\delta b y^{\sigma}$, where $\beta=\alpha+\bar{\alpha}$ and $\delta=\alpha-\bar{\alpha}$ as before. Now $c\left(\delta y+\beta b y^{\sigma}\right)^{\tau}=\beta y+\delta b y^{\sigma}$ for all $y \in L$. As in the proof of Lemma 1 this implies first that $c \delta^{\tau}\left(u^{\tau}-u^{\sigma \tau}\right) z^{\tau}=\beta\left(u-u^{\sigma \tau}\right) z+\delta b\left(u^{\sigma}-u^{\sigma \tau}\right) z^{\sigma}$ for all $u, z \in L$, and then that $\left.\tau\right|_{L}=\left.\sigma^{ \pm 1}\right|_{L}$.

Now suppose that $\left.\tau\right|_{L}=\left.\sigma\right|_{L}$ (cf. (I)). Then

$$
\left(c \delta^{\tau}-\delta b\right)\left(u^{\sigma}-u^{\sigma^{2}}\right) z^{\sigma}=\beta\left(u-u^{\sigma^{2}}\right) z \quad \text { for all } u, z \in L,
$$

which as before yields that $c \delta^{\tau}-\delta b=0$ and either $\beta=0$ or $\left.\sigma^{2}\right|_{L}=1$. Now $c \delta^{\tau} y^{\sigma}+c \beta^{\tau} b^{\tau} y^{\sigma \sigma}=\beta y+\delta b y^{\sigma}$ implies that $c \beta^{\tau} b^{\tau} y^{\sigma^{2}}=\beta y$. Then $c \beta^{\tau} b^{\tau}=\beta$, and either $\beta=0$ or $\left.\sigma^{2}\right|_{L}=1$. Since we already know that $c \delta^{\tau}=\delta b$, where $\delta^{\tau}=\delta^{\sigma}$ or $\delta^{\tau}=\bar{\delta}^{\sigma}=-\delta^{\sigma}$, one of the following holds:

$$
\begin{aligned}
& \beta=0, \delta \neq 0 \text { and } c= \pm \delta^{1-\sigma} b, \text { or } \\
& \beta \neq 0, c=\beta^{1-\sigma} b^{-\tau} \text { and }\left.\sigma^{2}\right|_{L}=1 .
\end{aligned}
$$

Since $g$ normalizes $\langle\tilde{t}\rangle$ it must send the $\langle\tilde{t}\rangle$-orbits on $\mathscr{S}_{b, \sigma}^{\prime}$ to those on $\mathscr{S}_{c, \tau}^{\prime}$. Thus, $\alpha h(b L)=t^{j} h^{\prime}(L)$ for some $j$; write $w=t^{j}$. Then

$$
w\left(x+c x^{\tau}\right)=\alpha\left(b y+b b^{\sigma} y^{\sigma}\right)
$$

for another permutation $x \rightarrow y$ of $L^{*}$.

Subcase 2.1: $\left.\sigma^{2}\right|_{L} \neq 1$. If $\tau=\sigma$ then $\delta^{\tau}=\delta^{\sigma}$, so that $c=\delta^{1-\sigma} b$ and $b$ lie in the same $H_{1}(\sigma)$-orbit, as required in (III). It remains to assume that $\tau=\sigma \theta$, or equivalently, $c=-\delta^{1-\sigma} b$, and derive a contradiction.

We have $\beta=0, \delta=\alpha-\bar{\alpha}=2 \alpha$ and $c \alpha^{\sigma}=-\alpha b$. By (4),

$$
w\left(x+c x^{\tau}\right)=\alpha\left(b y+b b^{\sigma} y^{\sigma}\right)=-c \alpha^{\sigma} y-c \alpha^{\sigma}\left(-c \alpha^{\sigma} / \alpha\right)^{\sigma} y^{\sigma}=v-c v^{\sigma}
$$


with $v=-c \alpha^{\sigma} y$. Here $v \in L$ since $\bar{\alpha}=-\alpha$. Now $k\left(x^{2}-c^{2} x^{2 \sigma}\right)=v^{2}-c^{2} v^{2 \sigma}$ where $k=w \bar{w}$ is a square in $K$. Then $k x^{2}-v^{2}=c^{2}\left(k x^{2}-v^{2}\right)^{\sigma}$ implies that, for some $m \in K$ and all $x \in L, v=m x$. Thus, $w\left(x+c x^{\sigma}\right)=m x-c m x^{\sigma}$, so that $w=m$ and $c w=-c m$, which is impossible.

Subcase 2.2: $\left.\sigma^{2}\right|_{L}=1$. As in Subcase 1.2 we may assume that $\tau=\sigma$. If $\delta \neq 0$ then $c=\delta^{1-\sigma} b$, and hence $b$ and $c$ are in the same $H_{1}(\sigma)$-orbit.

Assume that $\delta=0$, so that $\alpha \in L$ and $\alpha=c \alpha^{\sigma} b^{\sigma}$. By (4), $\bar{w}\left(x-c x^{\sigma}\right)=\alpha\left(-b y+b b^{\sigma} y^{\sigma}\right)$, so that $(w+\bar{w}) x+(w-\bar{w}) c x^{\sigma}=2 \alpha b b^{\sigma} y^{\sigma}$ and $(w-\bar{w}) x+(w+\bar{w}) c x^{\sigma}=2 \alpha b y$. Then

$$
\begin{aligned}
& \left\{(w+\bar{w}) x+(w-\bar{w}) c x^{\sigma}\right\} \alpha^{\sigma} \\
& \quad=2 \alpha b \alpha^{\sigma} b^{\sigma} y^{\sigma}=\alpha b\left\{(w-\bar{w}) x+(w+\bar{w}) c x^{\sigma}\right\}^{\sigma}
\end{aligned}
$$

for all $x \in K$. Consequently, $(w+\bar{w}) \alpha^{\sigma}=\alpha b(w+\bar{w})^{\sigma} c^{\sigma}$ and $(w-\bar{w}) c \alpha^{\sigma}=$ $\alpha b(w-\bar{w})^{\sigma}$. If $\bar{w} \neq w$ then $c=\{(w-\bar{w}) / \alpha\}^{\sigma-1} b$ with $(w-\bar{w}) / \alpha \in b L^{*}$, so that $b$ and $c$ are in the same $H_{1}(\sigma)$-orbit. Suppose that $\bar{w}=w$. Then $w^{2}=w \bar{w}$ is a square in $K$, so that $w \in K$. Now $\bar{w}=w=w^{\sigma}$, so that $(w+w) \alpha^{\sigma}=$ $\alpha b(w+w) c^{\sigma}$ and hence $\alpha^{\sigma}=\alpha b c^{\sigma}$. Then $\alpha=\alpha^{\sigma \sigma}=\alpha^{\sigma} b^{\sigma} c^{\sigma \sigma}=\alpha^{\sigma} b^{\sigma} \bar{c}=$ $-\alpha^{\sigma} b^{\sigma} c$, whereas we already knew that $\alpha=c \alpha^{\sigma} b^{\sigma}$.

Proof of Theorem 2, continued. (iii) Assume that there is a cyclic group acting transitively on the line at infinity. By Sylow's Theorem we may assume that $\tilde{s}_{0}$ lies in that cyclic group. Then that cyclic group is contained in $C_{\Gamma L(F)}\left(\tilde{s}_{0}\right)=\left\{\tilde{\alpha} \mid \alpha \in F^{*}\right\}$. In the notation of the proof of Lemma 2(III, IV) we have $b=c$, and there must be a collineation $g=\tilde{\alpha}$ of $\Pi_{b, \sigma}^{\prime}$ such that $\alpha h(L)=h(b L)$.

If $\left.\sigma^{2}\right|_{L} \neq 1$ then Case 2 of that proof yields that $\beta=0$ and $b=\delta^{1-\sigma} b$. Thus, $\delta^{\sigma}=\delta$ and $\delta=\alpha-\bar{\alpha} \notin L$. Consequently, the fixed field of $\sigma$ is not contained in $L$, which is not the case in (iii).

If $\left.\sigma^{2}\right|_{L}=1$ then we are in the situation of the proof of Lemma 2, Subcase 2.2 , where it was shown that $b=\delta^{1-\sigma} b$ or $b=\{(w-\bar{w}) / \alpha\}^{\sigma-1} b$. Then $\delta$ or $(w-\bar{w}) / \alpha$ lies in the fixed field of $\sigma$ but not in $L$, which is not the case.

(iv) As in the proof of Theorem 1(iii), if $\varphi$ is any power of $\sigma$ then $z \rightarrow\left(b^{(\varphi-1) /(\sigma-1)}\right)^{1-\sigma} z^{\varphi}$ fixed $b$, and moreover lies in $H_{1}(\sigma)_{b}$ since $b^{(\varphi-1) /(\sigma-1)} \in L^{*} \cup b L^{*}$. Thus, $\left|b^{H_{1}(\sigma)}\right| \leqslant\left|H_{1}(\sigma)\right| /|\sigma|$ for any $b$, so that the number of $H_{1}(\sigma)$-orbits on $b L^{*}$ is at least $|\sigma|\left(q^{n}-1\right) /\left(H_{1}(\sigma) \mid\right.$.

Let $n / n^{*}$ be the smallest prime factor of $n$. Choose $\sigma=q^{j n^{*}}$ where $1 \leqslant j n^{*}<n$ and $j$ is odd; note that no two of these automorphisms of $F$ are inverses of one another. In (iii) we require that the fixed field of $\sigma$ is contained in $L$, and this holds since $\left(j n^{*}, 2 n\right)=n^{*}$ is a factor of $n$ (recall that $j$ is odd). Note that $|\sigma|=2 n / n^{*}$, and

$$
\left|H_{1}(\sigma)\right|=4 e n\left(q^{n}-1\right) /\left(2\left(q^{n}-1\right), \sigma-1\right)=4 \operatorname{en}\left(q^{n}-1\right) /\left(q^{n^{*}}-1\right)
$$


since $\left.\left(q^{i n^{*}}-1\right) / q^{n^{*}}-1\right)$ is odd. Consequently, the number of $H_{1}(\sigma)$-orbits on $b L^{*}$ is at least $\left(2 n / n^{*}\right)\left(q^{n}-1\right) /\left|H_{1}(\sigma)\right|=\left(q^{n^{*}}-1\right) / 2 e n^{*}$.

If $n / n^{*}=2$ then this is precisely the assertion of (iv). If $n / n^{*}$ is odd then, by varying $j$, we obtain (iv).

(v) Moreover, if $n$ is prime then $n^{*}=1$ and $\sigma=q^{j}$ for some $j$ with $1 \leqslant j<2 n$. By Lemma 2(I) we may assume that $j \leqslant n$; and the requirement in (iii) that $(j, 2 n) \mid n$ forces $j$ to be odd. If, in addition, $q$ is prime, then $\left|H_{1}(\sigma)\right|=4 n\left(q^{n}-1\right) /(q-1) \quad$ and $\quad\left|b^{H_{1}(\sigma)}\right| \leqslant\left|H_{1}(\sigma)\right| /|\sigma|=2\left(q^{n}-1\right) /(q-1)$. However, there are $2\left(q^{n}-1\right) /(q-1)$ images of $b$ under the linear mappings $z \rightarrow \alpha^{1-\sigma_{z}} z$ with $\alpha \in L^{*} \cup b L^{*}$. Thus, $\left|b^{H_{1}(\sigma)}\right|=2\left(q^{n}-1\right) /(q-1)$ for each $b$, and hence $H_{1}(\sigma)$ has exactly $(q-1) / 2$ orbits on $b L^{*}$.

REMARKS. 1. Suppose that $n=n^{\prime} n^{\prime \prime}$ is the product of odd integers $n^{\prime}$, $n^{\prime \prime}>1$, and that $q^{n} \equiv 1(\bmod 4)$. Let $\sigma$ be the automorphism $q^{n^{\prime}}$ of $F$. Then the condition $\left.\sigma\right|_{L} \neq 1$ in Theorem 1 is certainly satisfied, and the fixed field of $\sigma$ is contained in $L$. By Theorem 2(iii), $\Pi_{b, \sigma} \nsucceq \Pi_{b, \sigma}^{\prime}$. Moreover, the spreads $\mathscr{S}_{b, \sigma}$ and $\mathscr{S}_{b, \sigma}^{\prime}$ share half of their members: some sort of 'net replacement' is at work here.

2. In Theorem 2 suppose that $n$ is odd but the fixed field of $\sigma$ is not contained in L. Claim: The spreads $\mathscr{S}_{b, \sigma}$ and $\mathscr{S}_{b, \sigma}^{\prime}$ coincide. Namely, let $\sigma=q^{r}$, so the fixed field of $\sigma$ is $\mathrm{GF}\left(q^{k}\right)$ where $k=(r, 2 n)$. Then $k \mid 2 n$ but $k \nmid n$, so that $2 n / k$ is odd. Let $u \in F^{*}$ have order $\left(q^{n}+1\right)\left(q^{k / 2}-1\right)$, let $j=\frac{1}{2}\left(q^{n}+1\right)$, and write $\alpha=u^{j}$. Since $\left(q^{n}-1\right) /\left(q^{k / 2}-1\right)$ is odd, $\alpha \notin L$ and $\alpha^{2} \in L$. Then $\alpha^{\sigma}=\alpha$, $\bar{\alpha}=-\alpha$, and $\alpha b, \alpha / b \in L$. It follows that $\alpha\left(x+b x^{\sigma}\right)=b(\alpha x / b)+b b^{\sigma}(\alpha x / b)^{\sigma}$ and $\alpha\left(b x+b b^{\sigma} x^{\sigma}\right)=(\alpha b x)+b(\alpha b x)^{\sigma}$, so that $\langle\tilde{u}\rangle$ is a collineation group transitive on the line at infinity of $\Pi_{b, \sigma}^{\prime}$. Since the kernel of $\Pi_{b, \sigma}^{\prime}$ contains $\operatorname{GF}\left(q^{k / 2}\right)$, we are in the situation of Theorem 1 but with $K$ replaced by $\mathrm{GF}\left(q^{k / 2}\right)$ and $s$ by $u$. This implies the Claim.

In particular, the Claim explains the restriction on the fixed field of $\sigma$ in Theorem 2(iii).

3. Theorem 2 allows the possibility that $2 n=4$, a case studied intensively by Baker and Ebert [1], [2]. I am indebted to Gary Ebert for pointing out that the construction in Theorem 2 - which is just Suetake's in that case (since we might as well let $\sigma=q$ by Lemma 2(I)) - settles the conjecture made on $\mathrm{p}$. 13 of [2] concerning the coordinate description of the planes studied there. The results in [1], [2] imply that every odd order nondesarguesian flagtransitive translation plane of dimension 2 over its kernel is one of the planes $\Pi_{b, q}^{\prime}$.

In [1] the authors examine the isomorphism problem for these planes. They use an unusual notion of isomorphism, viewing two translation planes as isomorphic if and only if there is a linear isomorphism between the planes; 
and then they conjecture that the number of pairwise nonisomorphic planes $\Pi_{b, q}^{\prime}$ is $(q-1) / 2$. This conjecture is precisely the content of Theorem 2(v) and its proof (when $n=2$ ), i.e., it is an immediate consequence of Lemma 2(II, IV). On the other hand, if $e>1$ is odd then it is easy to check that the number of planes is greater than $(q-1) / 2 e$.

4. If $G=\left\{z \rightarrow \alpha z^{\varphi}+u \mid \alpha \in F^{*}, u \in F, \varphi \in\right.$ Aut $\left.F\right\}$ then the proofs of Lemmas I and II easily imply that

$$
\begin{gathered}
G \cap \text { Aut } \Pi_{b, \sigma}=\left\{z \rightarrow \alpha z^{\varphi}+u \mid \alpha \in L^{*}, u \in F, \varphi \in \text { Aut } F, \text { and } \alpha^{\sigma-1}=b^{\varphi-1}\right\} \\
G \cap \text { Aut } \Pi_{b, \sigma}^{\prime}=\left\{z \rightarrow \alpha z^{\varphi}+u \mid \alpha \in L^{*} \cup b L^{*}, u \in F, \varphi \in \text { Aut } F,\right. \\
\text { and } \left.\alpha^{\sigma-1}=b^{\varphi-1}\right\} .
\end{gathered}
$$

However, quite a bit more group theoretic machinery seems to be needed in order to show that

Aut $\Pi_{b, \sigma}=G \cap$ Aut $\Pi_{b, \sigma}$ and Aut $\Pi_{b, \sigma}^{\prime}=G \cap$ Aut $\Pi_{b, \sigma}^{\prime}$ provided that $q^{n}>9$.

It would be nice to have an elementary proof of this fact.

5. In view of the constructions in [3] and [4], there exists at least one nondesarguesian flag-transitive affine plane of order $p^{n}$ whenever $p$ is a prime, $n>1, p^{n}>81$, and either $p$ is odd or $n$ is odd. It would be very interesting to know whether or not there exist such planes of order $2^{2 m}$ for any $m \geqslant 2$.

\section{REFERENCES}

1. Baker, R. D. and Ebert, G. L., 'Enumeration of two-dimensional flag-transitive planes', Algebras, Groups Geom. 3 (1985), 248-257.

2. Baker, R. D. and Ebert, G. L., 'Construction of two-dimensional flag-transitive planes', Geom. Dedicata 27 (1988), 9-14.

3. Kantor, W. M., 'Spreads, translation planes and Kerdock sets. II', SI AM J. Algebraic Discrete Methods 3 (1982), 308-318.

4. Suetake, C., 'Flag transitive planes of order $q^{n}$ with a long cycle on $l_{\infty}$ as a collineation' (to appear).

5. Wagner, A., 'On finite affine line transitive planes', Math. Z. 87 (1965), 1-11.

6. Zsigmondy, K., 'Zur Theorie der Potenzreste', Monatsh. Math. Phys. 3 (1892), 265-284.

Author's address:

W. M. Kantor,

Dept. of Mathematics,

University of Oregon,

Eugene, OR 97403-1222,

U.S.A.

(Received, April 1, 1990) 